\title{
p38 MAPK reins in right ventricular growth
}

\author{
Tongbin Wu and Ju Chen
}

Department of Medicine, UCSD, La jolla, California, USA.

\begin{abstract}
The right ventricle (RV) is involved in systemic circulation in the fetal mammalian heart but quickly transitions to being solely responsible for pulmonary circulation after birth when the left ventricle (LV) becomes the systemic ventricle. To handle the increased workload, LV growth greatly outpaces that of the RV during postnatal stages. However, the molecular basis for this differential growth pattern between the $\mathbf{2}$ chambers is largely unknown. In this issue of the $\mathrm{JCl}$, Yokota et al. reveal that the p38 mitogenactivated protein kinase (MAPK)/IRE1 $\alpha / \mathrm{XBP} 1$ axis specifically controls postnatal $\mathrm{RV}$ growth by suppressing cell cycle regulatory genes.
\end{abstract}

\section{Right versus left ventricle and related cardiomyopathies}

During the early stages of mammalian heart development, first-heart-field progenitors give rise to the left ventricle $(\mathrm{LV})$, while second-heart-field progenitors contribute to the right ventricle (RV) $(1,2)$. The LV and $\mathrm{RV}$ form a single chamber in the embryonic heart until they are separated by the interventricular septum (IVS) at E15 in mice and day 90 in humans (3). There is a dramatic shift in the role of the RV from fetal to postnatal stages. The fetal RV pumps blood to the lower body and placenta in addition to the lungs, whereas the postnatal RV is solely responsible for pulmonary circulation (4). Accordingly, the growth of the RV adapts to the low impedance of the pulmonary vasculature and maintains a relatively thin wall. In contrast, the growth of the LV accelerates to cope with increased pressure demands for pumping blood throughout the body, resulting in a much thicker LV wall (5). The rapid postnatal $\mathrm{LV}$ growth is at least partially due to its relatively high cardiomyocyte (CM) proliferation rate and low apoptosis rate compared with the RV (6). Nevertheless, the molecular network responsible for the differential growth pattern between the LV and RV is still largely unclear. It has been proposed that mechanical and envi- ronmental cues, such as blood volume and pressure $(5,7)$, may, in part, shape postnatal LV and RV morphologies. However, molecular mechanisms underlying the differential growth of the LV and RV, in particular chamber-specific cell-autonomous intracellular molecular pathways in CMs, remain to be determined. Although RV dysfunction is often interpreted as collateral damage consequent to severe $\operatorname{LV}$ dysfunction $(5,8)$, RV dysfunction itself can have profound adverse effects on patient outcome, leading to specific clinical manifestations such as pulmonary hypertension, arrhythmias, and premature death $(9,10)$. In addition, there are cardiomyopathies that specifically affect the RV. For instance, arrhythmogenic RV cardiomyopathy (ARVC) and RV noncompaction (RVNC) are cardiomyopathies that have contractile dysfunction and arrhythmias predominantly manifested in the RV $(11,12)$. Nevertheless, in spite of these intrinsic developmental and pathophysiological differences between RV and $\mathrm{LV}$, therapeutic options for RV dysfunction remain limited and often include treatments established for LV dysfunction (13).

In this issue of the JCI, Yokota and colleagues (14) sought to uncover signaling mechanisms specific to the RV by examining activities of mitogen-activated protein

Related Article: p. 5287

Conflict of interest: JC consulted for and receives research funding from MyoKardia Inc.

Copyright: () 2020, American Society for Clinical Investigation.

Reference information: / Clin Invest. 2020;130(10):5109-5111. https://doi.org/10.1172/JCI140793.

kinases (MAPKs) in the neonatal mouse heart. Surprisingly, the researchers found that p38 MAPK was specifically activated in the RV while remaining dormant in the LV, corresponding well with the lower CM proliferation observed in the RV, as p38 MAPK is known to suppress neonatal CM proliferation (15). To confirm that p38 MAPK indeed represses $\mathrm{CM}$ proliferation in the $\mathrm{RV}$, the authors went on to delete genes encoding p38 $\alpha$ and p38 $\beta$ (p38-cdKO) specifically in CMs. Postnatally, p38-cdKO mice developed RV-specific enlargement, associated with increased CM proliferation, increased CM hypertrophy, and decreased CM apoptosis in the RV. These mice progressed to develop pulmonary hypertension and right heart failure beyond the neonatal period without any discernible defects in their LVs. To further delineate underlying molecular mechanisms, Yokota et al. (14) compared $\mathrm{LV}$ and RV transcriptomes between p38cdKO and control mice. The authors discovered marked upregulation of cell cycle regulatory genes in both ventricles of mutants relative to controls, although differentially expressed genes (DEGs) in the RV of p38-cdKO mice tended to have much larger fold changes than those in the LV. These findings may partially explain why CM proliferation substantially increased in the RV but remained relatively normal in the LV of p38-cdKO mice. To identify potential downstream transcription factors (TFs) that regulate DEGs in p38-cdKO mice, the authors analyzed putative TF binding motifs at promoters of the RV-enriched DEGs, and identified a previously uncharacterized candidate, XBP1, in addition to the classic cell cycle-regulatory TF E2F. Interestingly, the XBP1 upstream activator IRE1 $\alpha$ was significantly upregulated in the $\mathrm{RV}$ of p38-cdKO mice. Consequently, the nucleus-localized, spliced XBP1 isoform (sXBP1), whose production is believed to be mediated by IRE1 $\alpha$ (16), was also upregulated only in the RV of p38-cdKO mice. To demonstrate that the p38 MAPK/IRE1 $\alpha$ / XBP1 pathway operates in CMs, Yokota et al. (14) turned to cultured neonatal rat ventricular myocytes (NRVMs). They found 
A

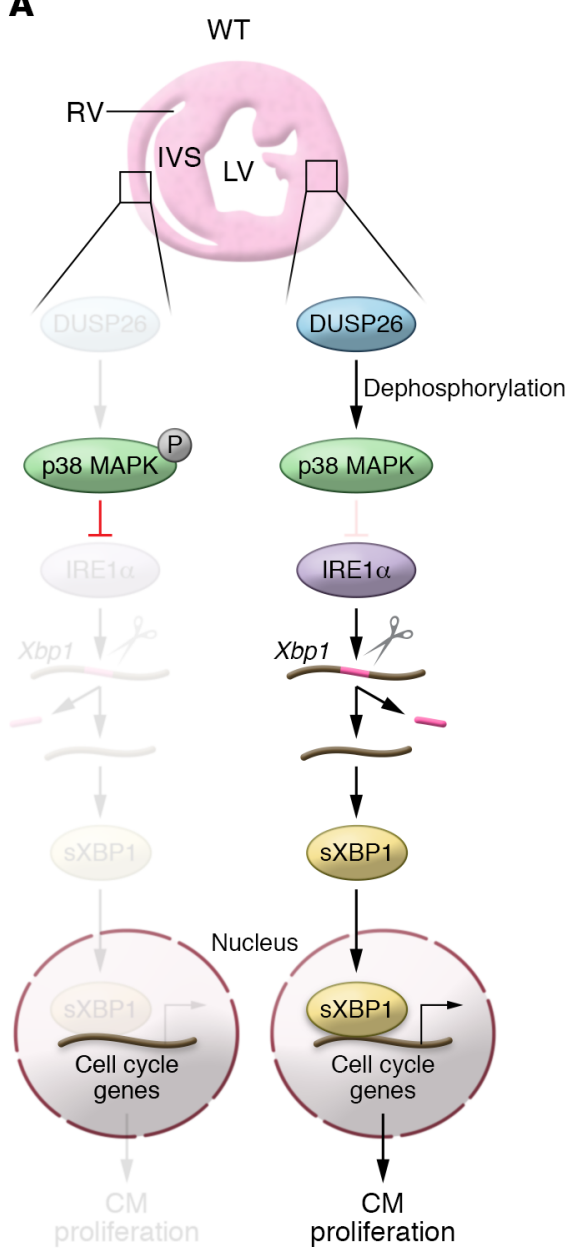

B

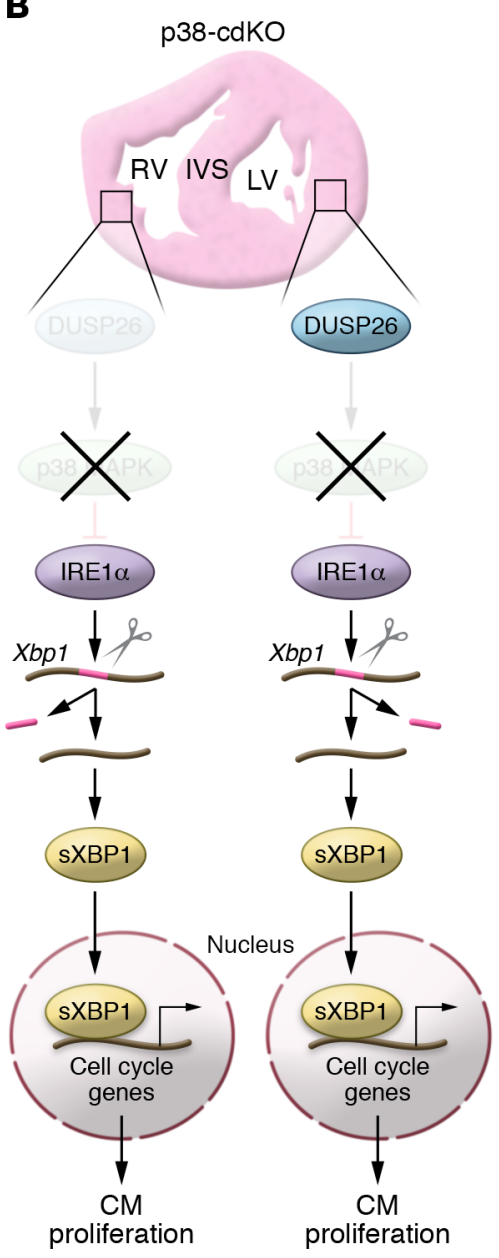

Figure 1. Model of DUSP26/p38 MAPK/IRE1 /XBP1 pathway in controlling cardiomyocyte (CM) proliferation in ventricles. (A) In the WT neonatal heart, low DUSP26 levels in the RV are insufficient to dephosphorylate 38 MAPK, which in turn represses the expression of IRE1 $\alpha$ and shuts down the IRE1 $\alpha / X B P 1$ pathway, leading to lower CM proliferation in the RV. In contrast, LV-enriched DUSP26 dephosphorylates p38 MAPK, thus relieving p38 MAPK's inhibitory effect on the expression of IRE1 $\alpha$, which splices Xbp1 mRNA to generate the $s$ Xbp1 isoform. sXBP1 then translocates to the nucleus and drives the expression of cell cycle-related genes, promoting CM proliferation. (B) In the p38-cdKO heart, the expression of IRE1 $\alpha$ in the RV is no longer repressed by $\mathrm{p} 38$ MAPK, resulting in ectopic activation of the IRE1 $\alpha$ /XBP1 pathway, which ultimately leads to excessive RV growth and dysfunction. In contrast, the p38-cdKO LV is unaffected because p38 MAPK activity is already very low in the WT LV.

that inhibiting p38 MAPK activity was sufficient to induce IRE1 $\alpha$ expression, increase expression of active SXBP1 relative to inactive, unspliced XBP1 ( $\mathrm{UXBP1}$ ), and enhance NRVM proliferation. In contrast, proliferation was attenuated in $\mathrm{p} 38 \mathrm{MAPK}$-inhibited NRVMs when XBP1 was knocked down, highlighting the essential role of the p38 MAPK/IRE1 $\alpha / \mathrm{XBP} 1$ pathway in controlling $\mathrm{CM}$ proliferation.

A remaining question was why $\mathrm{p} 38$ MAPK was specifically activated in the RV of neonatal mice. To address this question, the authors examined genes with chamberspecific expression patterns and found that a member of the dual-specific phosphatase family, DUSP26, had higher expression in the LV than in the RV. Knocking down DUSP26 in NRVMs led to significantly increased p38 MAPK activity and attenuated NRVM proliferation. These findings suggest that increased DUSP26 expression in the LV may result in greater inhibition of p38 MAPK in the LV than in the RV, leading to higher p38 MAPK activity in the RV.

\section{Unanswered questions and future directions}

This elegant study by Yokota and colleagues (14) provides a much-needed mouse model of RV dysfunction. Taking advantage of this model, the authors delineated a p38 MAPK/IRE1 $\alpha /$ XBP1 pathway that is specifically activated in the $\mathrm{RV}$ to limit CM proliferation (Figure 1A). In p38-cdKO mice, however, IRE1 $\alpha$ is no longer repressed by p38 MAPK, leading to ectopic activation of the IRE1 $\alpha / \mathrm{XBP} 1$ pathway and increased $\mathrm{CM}$ proliferation in the RV (Figure 1B). Although this study adds intriguing knowledge about RVspecific signaling pathways in the neonatal heart, these findings raise many questions. Biochemical assays might be needed to determine whether DUSP26 directly dephosphorylates p38 MAPK in vitro and in vivo, and in turn reduces its activity. It is also unclear whether additional chamberspecific phosphatases and/or kinases might act upstream of p38 MAPK.

IRE1 $\alpha / \mathrm{XBP1}$ is a well-established pathway in the cellular response to ER stress (17). Remarkably, Yokota and colleagues link this pathway to the regulation of CM proliferation in the RV (14). Although the expression of IRE1 $\alpha$ was specifically induced in the RV of p38cdKO mice, the molecular mechanism for this selective expression is unknown. As p38 MAPK is unlikely to directly regulate IRE1 $\alpha$ transcription, it might activate an intermediate transcriptional repressor(s) that in turn represses IRE1 $\alpha$ transcription. Identification of such IRE1 $\alpha /$ XBP1 pathway factors will provide additional targets to modulate $\mathrm{CM}$ proliferation in the RV.

Another fascinating question concerns the identification of direct downstream XBP1 targets that regulate the cell cycle. Although XBP1 consensus binding sequences were enriched in RV DEGs of p38-cdKO mice, whether XBP1 directly regulates these genes by targeted DNA binding remains to be determined. Although the authors inactivated XBP1 and found that inhibiting p38 failed to upregulate one of the putative cell cycle targets of XBP1, Ccnb2, it remains unclear whether Ccnb2 or other cell cycle-related genes that are dramatically upregulated in the RV of p38-cdKO mice are direct downstream target genes of XBP1. Further experiments using XBP1 ChIP-seq in CMs from the heart would be useful. On the other hand, cell cycle-related genes were also substantially upregulated 
in the LV, albeit to a lesser extent than observed in the RV. Are these genes also targeted by XBP1 in the LV? Since p38 MAPK was barely activated in the LV and expression of IRE1 $\alpha$ was unaffected in the LV of p38-cdKO mice, it is unlikely that cell cycle-related genes in the LV are regulated by the $\mathrm{p} 38 \mathrm{MAPK} / \mathrm{IRE} 1 \alpha / \mathrm{XBP} 1$ pathway. XBP1 ChIP-seq performed in the LV and RV could test the hypothesis that XBP1 targets differently between the 2 ventricles.

In Yakota et al. (14), mechanistic studies on the pivotal role of the p38 MAPK/IRE1 $\alpha$ / $\mathrm{XBP} 1$ axis in regulating $\mathrm{CM}$ proliferation were largely carried out with in vitro-cultured NRVMs. It remains to be seen whether these observations faithfully reflect the situation in vivo. It would be interesting to see whether ablating/reducing IRE1 $\alpha$ expression in p38-cdKO mice could attenuate the RV enlargement and dysfunction phenotype. Alternatively, administering IRE1 $\alpha$ inhibitors that specifically block sXBP1 splicing $(18,19)$ to p38-cdKO mice might demonstrate that the p38 MAPK/ IRE1 $\alpha / \mathrm{XBP} 1$ pathway plays a similar role in neonatal CMs in vivo as in NRVMs. If deemed effective in ameliorating RV phenotypes of p38-cdKO mice without detrimental effects on normal $\mathrm{LV}$ function, IRE1 $\alpha$ inhibitors might hold tremendous potential for treating RV abnormalities and dysfunction in human patients.

\section{Acknowledgments}

JC is funded by grants from the NHLBI and holds an American Heart Association Endowed Chair in Cardiovascular Research.

Address correspondence to: Ju Chen, Department of Medicine, UCSD, 9500 Gilman Drive, BSB 5025, Mail Code 0613C, La Jolla, California 92093, USA. Phone: 858.822.4276; Email: juchen@ucsd.edu.

1. Vincent SD, Buckingham ME. How to make a heart: the origin and regulation of cardiac progenitor cells. Curr Top Dev Biol. 2010;90:1-41.

2. Evans SM, Yelon D, Conlon FL, Kirby ML. Myocardial lineage development. Circ Res. 2010;107(12):1428-1444.

3. Bruneau BG. Signaling and transcriptional networks in heart development and regeneration. Cold Spring Harb Perspect Biol. 2013;5(3):a008292.

4. Rudolph AM. Congenital cardiovascular malformations and the fetal circulation. Arch Dis Child Fetal Neonatal Ed. 2010;95(2):F132-6.

5. Friedberg MK, Redington AN. Right versus left ventricular failure: differences, similarities, and interactions. Circulation. 2014;129(9):1033-1044.

6. Fernandez E, Siddiquee Z, Shohet RV. Apoptosis and proliferation in the neonatal murine heart. Dev Dyn. 2001;221(3):302-310.

7. Bishop SP. The myocardial cell: normal growth, cardiac hypertrophy and response to injury. Toxicol Pathol. 1990;18(4 pt 1):438-453.

8. Nucifora G, Aquaro GD, Masci PG, Pingitore A, Lombardi M. Magnetic resonance assessment of prevalence and correlates of right ventricular abnormalities in isolated left ventricular noncompaction. Am J Cardiol. 2014;113(1):142-146
9. Vonk-Noordegraaf A, et al. Right heart adaptation to pulmonary arterial hypertension: physiology and pathobiology. JAm Coll Cardiol. 2013;62(25 suppl):D22-D33.

10. Norozi K, et al. Incidence and risk distribution of heart failure in adolescents and adults with congenital heart disease after cardiac surgery. Am J Cardiol. 2006;97(8):1238-1243.

11. Corrado D, Link MS, Calkins H. Arrhythmogenic right ventricular cardiomyopathy. $N$ Engl J Med. 2017;376(1):61-72.

12. Ranganathan A, Ganesan G, Sangareddi V, Pillai AP, Ramasamy A. Isolated noncompaction of right ventricle--a case report. Echocardiography. 2012;29(7):E169-72.

13. Winter MM, et al. Latest insights in therapeutic options for systemic right ventricular failure: a comparison with left ventricular failure. Heart. 2009;95(12):960-963.

14. Yokota T, et al. p38 Mitogen-activated protein kinase regulates chamber-specific perinatal growth in heart. J Clin Invest. 2020;130(10):5287-5301.

15. Yokota T, Wang Y. p38 MAP kinases in the heart. Gene. 2016;575(2 pt 2):369-376.

16. Yoshida H, Matsui T, Yamamoto A, Okada T, Mori K. XBP1 mRNA is induced by ATF6 and spliced by IRE1 in response to ER stress to produce a highly active transcription factor. Cell. 2001;107(7):881-891.

17. Oakes SA, Papa FR. The role of endoplasmic reticulum stress in human pathology. Annu Rev Pathol. 2015;10:173-194.

18. Mimura N, et al. Blockade of XBP1 splicing by inhibition of IRE1 $\alpha$ is a promising therapeutic option in multiple myeloma. Blood. 2012;119(24):5772-5781.

19. Sheng $X$, et al. IRE1 $\alpha$-XBP1s pathway promotes prostate cancer by activating c-MYC signaling. Nat Commun. 2019;10(1):323. 